\title{
Schwanoma Maligno de Nervo Periférico Metastático para Pulmãoe Glândula Adrenal: Relato de Caso
}

doi: https://doi.org/10.32635/2176-9745.RBC.2021v67n4.1433

\author{
Metastatic Peripheral Nerve Malignant Schwannoma of Lungs and Adrenal Gland: Case Report \\ Schwannoma Maligno Metastásico de Nervio Periférico para Pulmones y Glándula Suprarrenal: Reporte de Caso
}

Maria Eduarda Turczyn De Lucca'; Leonardo Cesar Ferreira Antunes²; Jhulia Farinha Maffini³; Kaline Bubniakª ${ }^{4}$ Hanna Jeny Schimim5; Sue Hellen de Oliveira Munhos ${ }^{6}$; Leandro Carvalho Ribeiro7; Rosângela Stadnick Lauth de Almeida Torres ${ }^{8}$

\section{RESUM0}

Introduçáo: $\mathrm{O}$ schwanoma é um tumor pouco frequente que se origina das células de Schwann que recobrem os prolongamentos nervosos. A malignidade desses tumores é ainda mais rara, correspondendo a 5-10\% de todos os sarcomas. O prognóstico é reservado, mesmo após completa ressecção cirúrgica. Relato do caso: Paciente do sexo masculino, 79 anos, apresentou-se com lesão crostosa em perna esquerda com dois meses de evolução. A biópsia da lesão inicialmente revelou sarcoma pleomórfico, mas seu padrão imuno-histoquímico confirmou tumor maligno da bainha do nervo periférico. O tumor desenvolveu metástase linfonodal, pulmonar e adrenal dentro de poucos anos após o diagnóstico inicial. A ressecção do tumor adrenal não foi possível. O paciente desenvolveu síndrome paraneoplásica e teve piora do estado clínico, evoluindo a óbito. Conclusáo: Tendo em vista a forma inespecífica do aparecimento do tumor e sua agressividade, é importante ressaltar o papel da ressecção cirúrgica ampla no seu tratamento. Além disso, fica evidente a necessidade de novos relatos desse tipo de neoplasia para melhor definição de uma conduta mais apropriada, principalmente em casos de recorrência ou disseminação metastática. Palavras-chave: Neoplasias do Sistema Nervoso Periférico; Neurilemoma; Metástase Neoplásica; Glândulas Suprarrenais; Pulmão.

\section{ABSTRACT}

Introduction: Schwannoma is a rare type of tumor which grows from Schwann cells, that protect nerve extensions. The malignancy of these tumors is even rarer, corresponding to $5-10 \%$ of all sarcomas. The prognosis is dismal, even after complete surgical resection. Case report: A 79-year-old male patient presented with a crusted lesion in his left leg with two months of evolution. Initially, the biopsy of the lesion revealed pleomorphic sarcoma, but its immunohistochemical pattern was peripheral nerve sheath malignant tumor. The tumor developed lymph node, lung, and adrenal metastasis within a few years after the first diagnosis. Adrenal tumor resection was not possible. The patient developed paraneoplastic syndrome and his clinical status worsened, progressing to death. Conclusion: In view of the nonspecific form of tumor appearance and its aggressiveness, it is important to highlight the role of wide surgical resection in its treatment. In addition, it is clear that new case reports of this type of neoplasia are necessary for better definition of more appropriate conducts, especially in recurrence or metastatic cases.

Key words: Peripheral Nervous System Neoplasms; Neurilemmoma; Neoplasm Metastasis; Adrenal Glands; Lung.

\section{RESUMEN}

Introducción: El schwannoma es un poco común, el cual se origina en las células de Schwann, que cubren las extensiones nerviosas. La malignidad de estos tumores es aún más rara, y representa 5-10\% de todos los sarcomas. El pronóstico es reservado, incluso después de una resección quirúrgica completa. Relato del caso: Varón de 79 años que presenta una lesión costrosa en la pierna izquierda a los dos meses de evolución. La biopsia de la lesión reveló inicialmente un sarcoma pleomórfico, pero su patrón inmunohistoquímico era un tumor maligno de la vaina del nervio periférico. El tumor desarrolló metástasis en los ganglios linfáticos, pulmones y suprarrenales pocos años después del diagnóstico. La resección del tumor suprarrenal no fue posible. El paciente desarrolló síndrome paraneoplásico y empeoró el estado clínico, progresando hasta la muerte. Conclusión: Dada la forma inespecífica de aparición del tumor y su agresividad, es importante destacar el papel de la resección quirúrgica amplia en su tratamiento. Además, existe una clara necesidad de nuevos informes de este tipo de neoplasias para definir mejor un abordaje más adecuado, especialmente en casos de recurrencia o diseminación metastásica.

Palabras clave: Neoplasias del Sistema Nervioso Periférico; Neurilemoma; Metástasis de la Neoplasia; Glándulas Suprarrenales; Pulmón.

\footnotetext{
1-4,8Universidade Positivo. Curitiba (PR), Brasil.E-mails: mariatdelucca@gmail.com; leonardocfa@hotmail.com; jhuliamaffini@gmail.com; kalinebubniak@gmail.com; rslatorres@gmail.com. Orcid iD: https://orcid.org/0000-0002-7625-8193; Orcid iD: https://orcid.org/0000-0002-3963-3716; Orcid iD: https://orcid.org/00000003-0930-7087; Orcid iD: https://orcid.org/0000-0002-9095-6166; Orcid-iD: https://orcid.org/0000-0002-8974-3323

5-7Hospital Erasto Gaertner. Curitiba (PR), Brasil. E-mails: hanna.schimim@hotmail.com; suemunhos86@gmail.com; lecribeiro@hotmail.com. Orcid iD: https:// orcid.org/0000-0002-1523-1296; Orcid iD: https://orcid.org/0000-0001-9557-2789; Orcid iD: https://orcid.org/0000-0002-9128-7021

Endereço para correspondência: Maria Eduarda Turczyn De Lucca. Hospital Erasto Gaertner. Rua Doutor Ovande do Amaral, 201 - Jardim das Américas. Curitiba (PR), Brasil. CEP 81520-060. E-mail: mariatdelucca@gmail.com
} 


\section{INTRODUÇÃO}

O schwanoma é um tumor raro, oriundo das células de Schwann que compóem a bainha dos nervos periféricos. Sua transformação maligna corresponde a apenas 5 a $10 \%$ de todos os sarcomas ${ }^{1-3}$. Em 1935, a neoplasia foi primeiramente interpretada como um fibrossarcoma e secundariamente - em 1949 - foi observado o crescimento do tumor a partir das células de Schwann ${ }^{4,5}$. Apesar disso, a histogênese do tumor é pouco conhecida e então outras denominaçóes são dadas: tumor maligno da bainha do nervo periférico, sarcoma neurogênico e neurofibrossarcoma ${ }^{2}$.

Os schwanomas malignos solitários são tumores raros e afetam principalmente o sexo masculino. Podem se desenvolver em qualquer idade, mas o pico de incidência ocorre entre 20 e 50 anos, frequentemente em nervos periféricos de maior diâmetro, podendo manifestar sintomas de neuropatia periférica ${ }^{4-7}$. Os sítios mais afetados são o pescoço, antebraço, pernas e nádegas. Esse tumor é frequentemente associado à neurofibromatose tipo I e extremamente raro em pacientes náo portadores dessa condição. O diagnóstico é influenciado pela apresentação clínica, e deve ser confirmado pela imuno-histoquímica ${ }^{6}$.

$\mathrm{O}$ tratamento é cirúrgico por não responder bem à quimioterapia ou à radioterapia convencional. Alguns estudos têm demonstrado remissão do tumor associando quimioterápicos com adriamicina ${ }^{8}$. Seu prognóstico é reservado, mesmo quando ressecado completamente. Metástases ocorrem principalmente em pulmóes, omento, cólon sigmoide e bexiga urinária ${ }^{1,2,5,6}$. Não existem recomendaçóes na literatura a respeito do manejo adequado em caso de recorrência ${ }^{6}$.

O presente trabalho visa a relatar um caso de schwanoma maligno de nervo periférico com metástases para glândula adrenal e pulmão. $\mathrm{O}$ estudo foi aprovado pelo Comitê de Ética em Pesquisa da Liga Paranaense de Combate ao Câncer do Hospital Erasto Gaertner sob protocolo CAAE: 87548518.2.0000.0098.

\section{RELATO DO CASO}

Paciente do sexo masculino, 79 anos, com hipertensão arterial sistêmica, insuficiência aórtica moderada e depressão, relata ter surgido dois meses antes da consulta com pequena lesão na regiáo anterior do terço inferior da perna esquerda, inicialmente na forma de crosta evoluindo para lesáo extensa e hiperemiada. Ao exame, identificou-se lesão crostosa de cerca de $2 \mathrm{~cm}$, com área de hiperemia circundante com aproximadamente $6 \mathrm{~cm}$. A biópsia da lesão inicialmente revelou sarcoma pleomórfico. Sua análise imuno-histoquímica é mostrada na Tabela 1A. O quadro histológico e o perfil imuno-histoquímico apontaram um tumor maligno da bainha do nervo periférico. O paciente não era portador de neurofibromatose tipo I.

Tabela 1. Painel imuno-histoquímico das lesões ressecadas. A. marcadores da biópsia do tumor primário. B. marcadores do produto de linfadenectomia. C. marcadores do produto de ressecção de nódulo pulmonar. D. marcadores do produto de adrenalectomia

\begin{tabular}{|c|c|c|c|c|}
\hline Marcador & $\begin{array}{l}\text { (A) } \\
\text { Biópsia tumoral }\end{array}$ & $\begin{array}{c}\text { (B) } \\
\text { Produto de } \\
\text { linfadenectomia inguinal }\end{array}$ & $\begin{array}{c}\text { (C) } \\
\text { Nódulo pulmonar }\end{array}$ & $\begin{array}{l}\text { (D) } \\
\text { Lesão } \\
\text { adrenal }\end{array}$ \\
\hline Alfa-AML & $\begin{array}{l}\text { Positivo multifocal nas } \\
\text { células neoplásicas }\end{array}$ & Negativo & Negativo & Negativo \\
\hline Vimentina & Positivo forte e difuso & Positivo difuso & $\begin{array}{c}\text { Positivo nas células } \\
\text { neoplásicas }\end{array}$ & Positivo \\
\hline$S-100$ & $\begin{array}{c}\text { Positivo nas células } \\
\text { neoplásicas }\end{array}$ & $\begin{array}{l}\text { Positivo nas células } \\
\text { pleomórficas }\end{array}$ & $\begin{array}{c}\text { Positivo nas células } \\
\text { neoplásicas }\end{array}$ & - \\
\hline CD-68 & $\begin{array}{l}\text { Positivo multifocal nas } \\
\text { células neoplásicas }\end{array}$ & Positivo nos histiócitos & 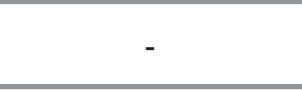 & Positivo focal \\
\hline Ki-67 & $\begin{array}{c}\text { Positivo em } 80 \% \text { dos } \\
\text { núcleos das células } \\
\text { neoplásicas }\end{array}$ & $\begin{array}{l}\text { Positivo em } 60 \% \text { dos núcleos } \\
\text { das células neoplásicas }\end{array}$ & $\begin{array}{l}\text { Positivo em } 70 \% \\
\text { dos núcleos das } \\
\text { células neoplásicas }\end{array}$ & - \\
\hline HMB-45 & Negativo & Negativo & - & - \\
\hline Desmina & Negativo & - & - & - \\
\hline CD-34 & Negativo & - & - & - \\
\hline CKAE 1/AE3 & Negativo & - & - & - \\
\hline MYO D1 & Negativo & - & - & - \\
\hline Melan A & Negativo & - & - & - \\
\hline PAX-8 & - & - & - & Negativo \\
\hline
\end{tabular}


Dois meses após o diagnóstico, foi realizada ressecção ampla do tumor da perna esquerda com enxertia. $\mathrm{O}$ exame anatomopatológico revelou: lesão de $2,5 \times 2 \times$ $0,9 \mathrm{~cm}$ de aspecto ulcerado, crostoso, vegetante e mal delimitado com características de neoplasia fusocelular ulcerada em pele. As tomografias computadorizadas (TC) de tórax e de pelve de controle náo apresentaram anormalidades. Por meio da TC de abdome, foram identificados pequenos nódulos hepáticos hipodensos, medindo 9 e $7 \mathrm{~mm}$ e um foco de calcificação de $7 \mathrm{~mm}$. A análise imuno-histoquímica do material enviado ao exame anatomopatológico revelou o mesmo padráo observado na biópsia (Figura 1) (Tabela 1A). Tendo em vista o predomínio absoluto de áreas pleomórficas na histologia tumoral, áreas Antoni A e Antoni B, bem como corpos de Verocay, não foram encontradas com nitidez na amostra de neoplasia.

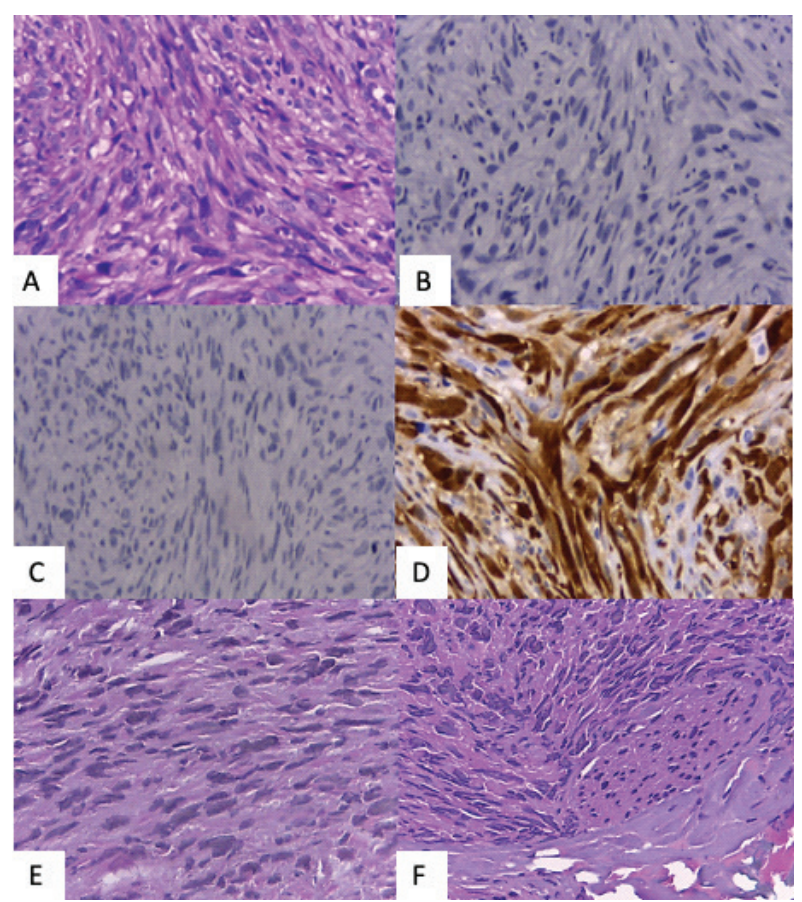

Figura 1. Histologia e imuno-histoguímica do tumor primário. A. Corte histológico contendo neoplasia composta por células fusiformes variavelmente pleomórficas formando fascículos e estruturas estoriformes com atividade mitótica presente. B. Ausência de imunorreatividade para Melan-A. C. Ausência de imunorreatividade para HMB-45. D. Presença de imunorreatividade para S-100. E. Corte histológico com células atípicas fusiformes, arranjadas em feixes, com limites citoplasmáticos indistintos e citoplasmas finalmente fibrilares e ondulados, conforme padrão de fibras nervosas. F. Corte histológico com células neoplásicas constituindo esboços de fibras neurais

Um ano após a ressecção, foi realizada punção aspirativa por agulha fina para investigação de linfonodomegalia na região inguinal esquerda. A análise histológica foi positiva para malignidade. Foi proposto o esvaziamento linfonodal inguinocrural esquerdo, cujo exame anatomopatológico revelou neoplasia pouco diferenciada, infiltrativa, em um dos quinze linfonodos analisados. $\mathrm{O}$ quadro histológico e o perfil imuno-histoquímicos eram condizentes com schwanoma maligno. Os marcadores imuno-histoquímicos obtidos são mostrados na Tabela 1B.

$\mathrm{Na}$ primeira TC de tórax (Figura 2A), realizada um ano e oito meses após o diagnóstico de neoplasia, foi evidenciado um novo nódulo de $21 \mathrm{~mm}$ no segmento posterior do lobo inferior do pulmáo esquerdo, sem calcificaçôes, levantando-se a hipótese de metástase pulmonar. Uma nova TC de tórax (Figura 2B), três meses depois, mostrou crescimento do nódulo para 31 $\mathrm{mm}$. Foi realizada a segmentectomia pulmonar esquerda com congelação intraoperatória que confirmou metástase (foco de $4 \mathrm{~cm}$ ). $\mathrm{O}$ anatomopatológico da lesão mostrou neoplasia pouco diferenciada com células pleomórficas e extensa necrose. Seu padrão imuno-histoquímico é observado na Tabela $1 \mathrm{C}$.

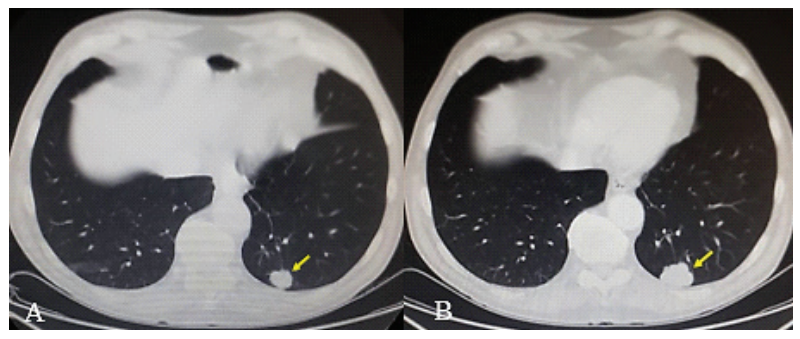

Figura 2. Nódulo pulmonar evidenciado primariamente em $\mathrm{A}$ e sua evolução em B

Um ano após a ressecção, o exame de ressonância magnética (RM) mostrou surgimento de massa adrenal à esquerda (Figura 3), caracterizada como lesão expansiva de contornos irregulares e realce predominantemente periférico com restrição à difusão na sua porção periférica, medindo 65 × $35 \mathrm{~mm}$. Nas suas porções centrais, identificou-se conteúdo necrótico. Neste momento, o paciente foi hospitalizado por episódios de diarreia, vômitos, febre e piora importante da função renal.

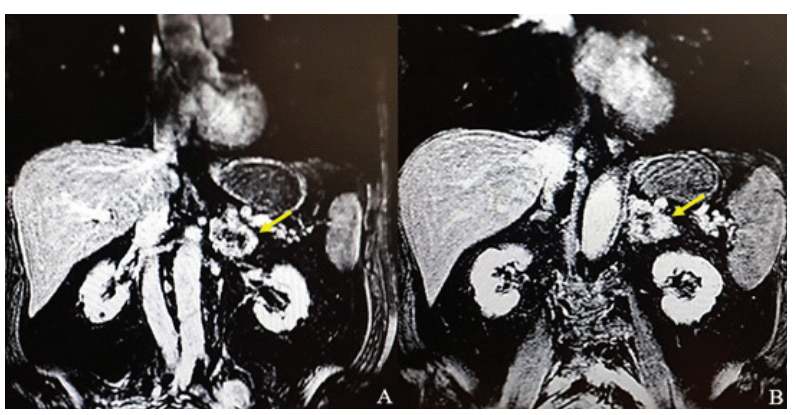

Figura 3. Massa adrenal à esquerda

No mês seguinte, uma nova TC de tórax revelou linfonodomegalia infracarinal, nódulo alongado associado a faixas de atelectasia no segmento basal posterior do lobo 
inferior esquerdo e nódulos com contornos lobulados medialmente no lobo inferior direito. Realizou-se a exenteraçáo do quadrante adrenal superior esquerdo, pela ausência de plano de clivagem com aorta, veia renal esquerda e hilo esplênico. Houve lesão do parênquima esplênico com hemostasia ineficaz por videolaparoscopia, havendo necessidade de esplenectomia. Durante o tempo de Unidade de Terapia Intensiva (UTI), o paciente não desenvolveu infecção.

O exame anatomopatológico da lesão adrenal revelou neoplasia pouco diferenciada e imuno-histoquímica compatível com sarcoma pleomórfico de alto grau (Tabela 1D) (Figura 4). Evoluiu no pós-operatório com hiporexia, náuseas e perda ponderal de cerca de $10 \mathrm{~kg}$. Nova TC de tórax revelou nódulos metastáticos esparsos em ambos os pulmóes (o maior deles com $21 \mathrm{~mm}$ ) e aumento da linfonodomegalia mediastinal. A TC de abdome mostrou massa sólida em glândula adrenal esquerda medindo 86 x 69 x $67 \mathrm{~mm}$, envolvendo parcialmente a veia e a artéria renal esquerda, fazendo contato com a aorta, pâncreas e rim esquerdo.

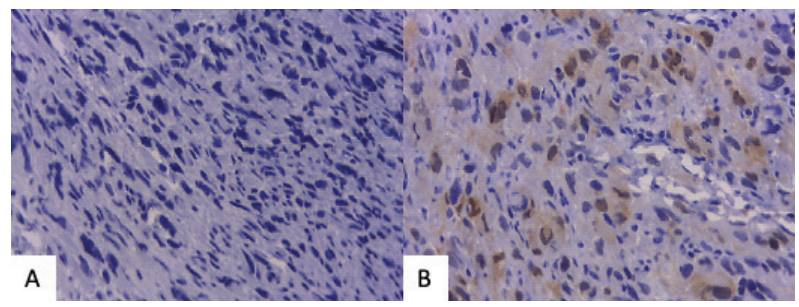

Figura 4. Imuno-histoquímica da metástase para adrenal. A. Ausência de imunorreatividade para Melan-A em neoplasia metastática composta por células fusiformes variavelmente pleomórficas. B. Presença de imunorreatividade para S-100

Pelas condiçôes clínicas do paciente, não houve indicação de quimioterapia, entrando em cuidados paliativos exclusivos. Alguns meses depois, internou para controle de hipercalcemia por síndrome paraneoplásica (tratamento com ácido zoledrônico). Apresentou diminuição da capacidade de deambulação, dispneia, anorexia e queda importante do estado clínico, evoluindo a óbito.

\section{DISCUSSÃO}

Schwanoma maligno com metástase para a glândula adrenal ainda não havia sido relatada na literatura. $\mathrm{O}$ paciente deste caso apresentava um tumor maligno, agressivo, mas não era portador de neurofibromatose tipo I. A presença de metástases adrenais é, por si só, um fator de mau prognóstico, com sobrevida em cinco anos de $20 \%$.

Os tumores que mais frequentemente causam metástases adrenais são o de pulmão e o de mama. Em virtude da escassez de estudos a respeito do assunto, o tratamento cirúrgico costuma ser empregado. Ele deve ser indicado na vigência de controle ou plano de controle da doença extra-adrenal, presença de metástases isoladas e confinadas em uma ou ambas as glândulas, imaginologia altamente sugestiva ou comprovação por exame patológico e estado funcional que possam permitir a intervenção cirúrgica9.

Esse tipo de tumor não apresenta sintomas específicos ou critérios diagnósticos bem definidos (exceto se tumor claramente conectado a um nervo ou associado à neurofibromatose tipo I ${ }^{10}$. Geralmente se apresenta como um nódulo fixo de crescimento lento, e sua pesquisa pode revelar a origem nervosa do tumor ${ }^{4}$.

Os schwanomas malignos frequentemente produzem metástases hematológicas e os sítios mais comuns são os pulmóes, o omento, o cólon sigmoide e a bexiga urináriaa $^{1,2,5,6}$. Metástases linfonodais são raras. Dois dos casos estudados por White ${ }^{1}$ apresentaram metástases linfonodais, mas nenhuma foi encontrada em 31 pacientes com schwanoma maligno estudados por Vieta e Pack ${ }^{11}$. Das Gupta e Brasfield ${ }^{12}$ não relataram nenhuma metástase linfonodal em 232 casos de schwanomas malignos solitários.

A angiografia auxilia em uma melhor abordagem terapêutica ao avaliar a vascularização do tumor, origens do suprimento e suas relações anatômicas ${ }^{5}$. As características radiológicas do tumor ainda não são definidas ${ }^{2}$. $\mathrm{O}$ tratamento da doença é cirúrgico por oferecer melhor possibilidade de cura ou cuidados paliativos a longo prazo. Radioterapia ou quimioterapia não tiveram sucesso, exceto entre os pacientes que fizeram uso de combinações com adriamicina ${ }^{1,5,10}$.

A ressecção completa é essencial, sendo recomendável também, além dos tecidos adjacentes, a ressecção de um longo segmento de nervo proximal ao tumor e sua análise por congelação intraoperatória, tendo em vista relatos de casos com disseminaçấo local do tumor. Alguns autores defendem que o tratamento de escolha para os tumores com crescimento nodular em nervo periférico seja a amputação, argumentando maior sobrevida do que realizar excisão local ${ }^{1,5,6,10}$. Em um estudo de 1952, estimou-se que $30 \%$ dos casos operados tiveram sobrevida maior ou igual a cinco anos ${ }^{1}$. Já em um estudo de 1981, relatou-se que a sobrevida entre pacientes com schwanoma maligno e neurofibromatose tipo I foi significativamente inferior (23\% em cinco anos) à de pacientes com schwanoma maligno isolado ( $47 \%$ em cinco anos). A maior parte dos óbitos entre os portadores da doença ocorreu nos dois primeiros anos de manifestação do tumor ${ }^{8}$.

O prognóstico de tumores paravertebrais é pior do que o de outras localizaçóes por geralmente se apresentarem em estádios avançados, envolvendo vísceras adjacentes ${ }^{4}$. Outro fator de pior prognóstico é a associação do tumor com a neurofibromatose tipo I, uma vez que as lesóes são 
mais pleomórficas e menos frequentemente encontradas em extremidades inferiores ${ }^{4,5,8}$. O prognóstico é ainda pior entre os pacientes com metástases pulmonares: apenas três de 71 pacientes estudados por Yamakami et al. ${ }^{2}$ sobreviveram por mais de dois anos após a descoberta da disseminação. Os tumores com menos de $5 \mathrm{~cm}$ de diâmetro e localizados nas extremidades, distantes das regióes axilar e inguinal, quando submetidos ao tratamento adequado possuem melhor prognóstico ${ }^{2,5}$.

Formaçóes osteoides e cartilaginosas já foram notadas nessa patologia ${ }^{4}$. O desenvolvimento desse tumor é caracterizado por hipocelularidade, pleomorfismo celular/ nuclear, presença de mitoses e proliferação de pequenos vasos, o que o assemelha aos fibrossarcomas ${ }^{3}$. A presença de achados de morfologia neural contribui grandemente para a hipótese. A negatividade do marcador HMB-45 também corrobora o diagnóstico do schwanomas, tal como ocorreu no presente caso relatado, e a positividade da proteína $S 100$ confirma o diagnóstico ${ }^{13}$.

\section{CONCLUSÃO}

Os schwanomas malignos são neoplasias raras de quadro clínico inespecífico e prognóstico sombrio, geralmente estão associados à neurofibromatose tipo I. O presente relato não tem relação com essa doença e é o primeiro na literatura a apresentar metástase adrenal, sendo sua importância também relacionada à necessidade de mais casos descritos sobre o assunto tanto para elucidar as características do tumor como para definir o manejo mais adequado.

\section{CONTRIBUIÇÕES}

Os autores contribuíram de forma equivalente em todas as etapas do trabalho, assim como aprovaram a versão a ser publicada.

\section{DECLARAÇÃO DE CONFLITO DE INTERESSES}

Nada a declarar.

\section{FONTES DE FINANCIAMENTO}

Não há.

\section{REFERÊNCIAS}

1. White HR Jr. Survival in malignant schwannoma. An 18-year study. Cancer. 1971;27(3):720-9. doi: https:// doi.org/10.1002/1097-0142(197103)27:3<720::AIDCNCR2820270331>3.0.CO;2-D
2. Yamakami I, Oishi $\mathrm{H}$, Iwadate $Y$, et al. Isolated metastases of adenocarcinoma in the bilateral internal auditory meatuses mimicking neurofibromatosis type 2--case report. Neurol Med Chir (Tokyo). 1999;39(11):756-61. doi: https://doi.org/10.2176/nmc.39.756

3. Viola E, Solà M, Stroppa S, et al. Malignant schwannoma in the posterior tibial nerve. Foot. 2006;16(4):216-7. doi: https://doi.org/10.1016/j.foot.2006.07.008

4. Ghosh BC, Ghosh L, Huvos AG, et al. Malignant schwannoma. A clinicopathologic study. Cancer. 1973;31(1):184-90. doi: https://doi. org/10.1002/1097-0142(197301)31:1<184::AIDCNCR2820310126>3.0.CO;2-8

5. Fabbro MA, Costa L, D'Agostino S, et al. Juxta-adrenal malignant schwannoma. Pediatr Surg Int. 1997;12:5324. doi: https://doi.org/10.1007/BF01258720

6. Rayatt SS, Jones ME, Powell BW. Surgical treatment of locally recurrent malignant schwannoma of the lower leg. Br J Plast Surg. 1999;52(4):317-9. doi: https://doi. org/10.1054/bjps.1999.3109

7. Wood MK, Erdmann MW, Davies DM. Malignant schwannoma mistakenly diagnosed as carpal tunnel syndrome. J Hand Surg Br. 1993;18(2):187-8. doi: https://doi.org/10.1016/0266-7681(93)90104-n

8. Sordillo PP, Helson L, Hajdu SI, et al. Malignant schwannoma - clinical characteristics, survival, and response to therapy. Cancer. 1981;47(10):2503-9. doi: https://doi. org/10.1002/1097-0142(19810515)47:10<2503::AIDCNCR2820471033>3.0.CO;2-3

9. Serra C. Metástases suprarrenais. Rev Port Cir [Internet]. 2015;32:27-34 [acesso 2020 out 26]. Disponível em: http://www.scielo.mec.pt/scielo.php?script=sci_ arttext\&pid=S1646-69182015000100005\&lng=pt

10. Herness D, Posner MA, Steiner G. Malignant schwannoma. Hand. 1975;7(3):300-2. doi: https://doi. org/10.1016/0072-968X_75_90074-1

11. Vieta JO, Pack GT. Malignant neurilemomas of peripheral nerves. Am J Surg. 1951;82(4):416-31. doi: https://doi.org/10.1016/0002-9610(51)90368-6

12. Das Gupta TK, Brasfield RD. Solitary malignant schwannoma. Ann Surg. 1970;171(3):419-28. doi: https://doi.org/10.1097/00000658-19700300000016

13. Pádua SC, Soares VYR, Queiroz AL, et al. Schwannoma maligno dos seios paranasais. Braz J Otorhinolaryngol. 2012;78(4):135. doi: https://doi.org/10.1590/S180886942012000400026 